\title{
Dynamical Modelling and Parameters Identification of Real Power Plant Synchronous Generator and Excitation System
}

\author{
Abolfazl Khodadadi* Behrooz Zaker* Ramtin Khalili* Seyed Mehdi Hosseini* Shahab Karrari* Mehdi Karrari* \\ *Electrical Engineering Department, Amirkabir University of Technology, Tehran, Iran \\ (Emails: \{a.khodadadi, zaker.behrooz, ramtin.khalili,sm-hosseini, shkarrari, karrari\}@aut.ac.ir)
}

\begin{abstract}
This paper presents a mathematical modelling and parameter identification for the excitation system with detailed model of comprising cards and synchronous generator. Then, the parameters of overall model are identified using an optimization algorithm. Data for identification process is obtained from online measurements of a $154 \mathrm{MW}$ gas power plant which is acquired from a data acquisition system. The validation process is carried out in Power Factory DIgSILENT software and the proper matching between simulation results and measurements data demonstrates the accuracy of the proposed model.
\end{abstract}

Keywords: electric power systems, parameter identification, close-loop identification, excitation systems, synchronous generator.

\section{INTRODUCTION}

Due to the inaccessibility to all of the power plant documents, having the mismatch between the existent documents and real system and altering the dynamic and static parameters with the passage of time, the dynamic parameters identification is essential for predicting the behavior of overall power network in the transient analyses. Even if the manufacturer proposed the as-built parameters, the validity of these parameters should be verified via field tests after five years. In the synchronous generator section, there are two identification methods: 1) offline identification and 2) online identification. In the offline methods, the synchronous generator is out of service and various techniques like frequency response, least squares, and dc excitation ones will be employed. In online methods a small exogenous signal is injected to the in-service machine in order to have no dominant effect on the normal operation of the system.

Generally, the direct method of excitation system identification necessitates the availability of excitation current and voltage which is not accessible in brushless excitation systems. However, the comprehensive identification of synchronous generator parameters makes the direct method possible in the brushless excitation systems.(Zaker et al., (2016))

In this paper, a mathematical modeling and parameter identification of synchronous generator and excitation system for a real gas power plant are presented. In this model, the effective parameters are identified using imperialistic competitive algorithm (ICA). The identified parameters are put in a general single synchronous generator and excitation system framework in the Power Factory DIgSILENT Software and the simulation results are compared with experimental results measured from a real 154MW gas power plant.

\section{GENERATOR MODELLING}

In this section, the seventh-order model of synchronous generator is considered. The advantage of this model is to consider the impacts of stator and damper windings comparing to the low-order linear structure such as Heffron-Philips. As a general role for synchronous generator modeling in a single machine system, flux linkages are chosen as the state variables. As it was stated before, this excitation system is a brushless type in which there is no way to measure the synchronous generator excitation current and voltage. Therefore, indirect method should be employed for the identification process.

\section{EXCITATION MODELLING}

This section consists of various parts each of which plays an important role in the overall performance of the system. The understudy excitation system is an alternator-supplied rectifier which employs ac alternator to produce dc current for the main generator field winding. In this section, the model of each card within the excitation system with a short description on each one is presented.

3.1 Main Regulator Section (RS Card)

This is the main section of automatic excitation system in which by implementing two types of controller, PI and PID, the regulation and stability functions are addressed.

3.2 Under-Excitation Detection (LSES Card)

This device prevents the de-magnetization of the generator when absorbing reactive power, and maintains the generator within its operating limits.

3.3 Over-Flux Section (LUF2 Card)

This card provides the U/f image of a three-phase system which is related to the excitation system flux, and the difference between this voltage image and an adjustable reference voltage $(\Delta \mathrm{U} / \mathrm{f})$.

3.4 Power System Stabilizer (SP1 Card)

This card provides the RS regulation card with a stabilizing signal as a function of the generator active power deviations. In fact, the oscillatory nature of the active power signal contains dynamical modes which their inclusion in the AVR section can enhance the power system dynamic performance. 3.5 Pulse Generation Section (GITS Card)

This section elaborates the control pulses for the thyristor bridge. The output of this bridge is connected to the exciter field windings. The result is a rectified voltage proportional to the control voltage of the AVR. 


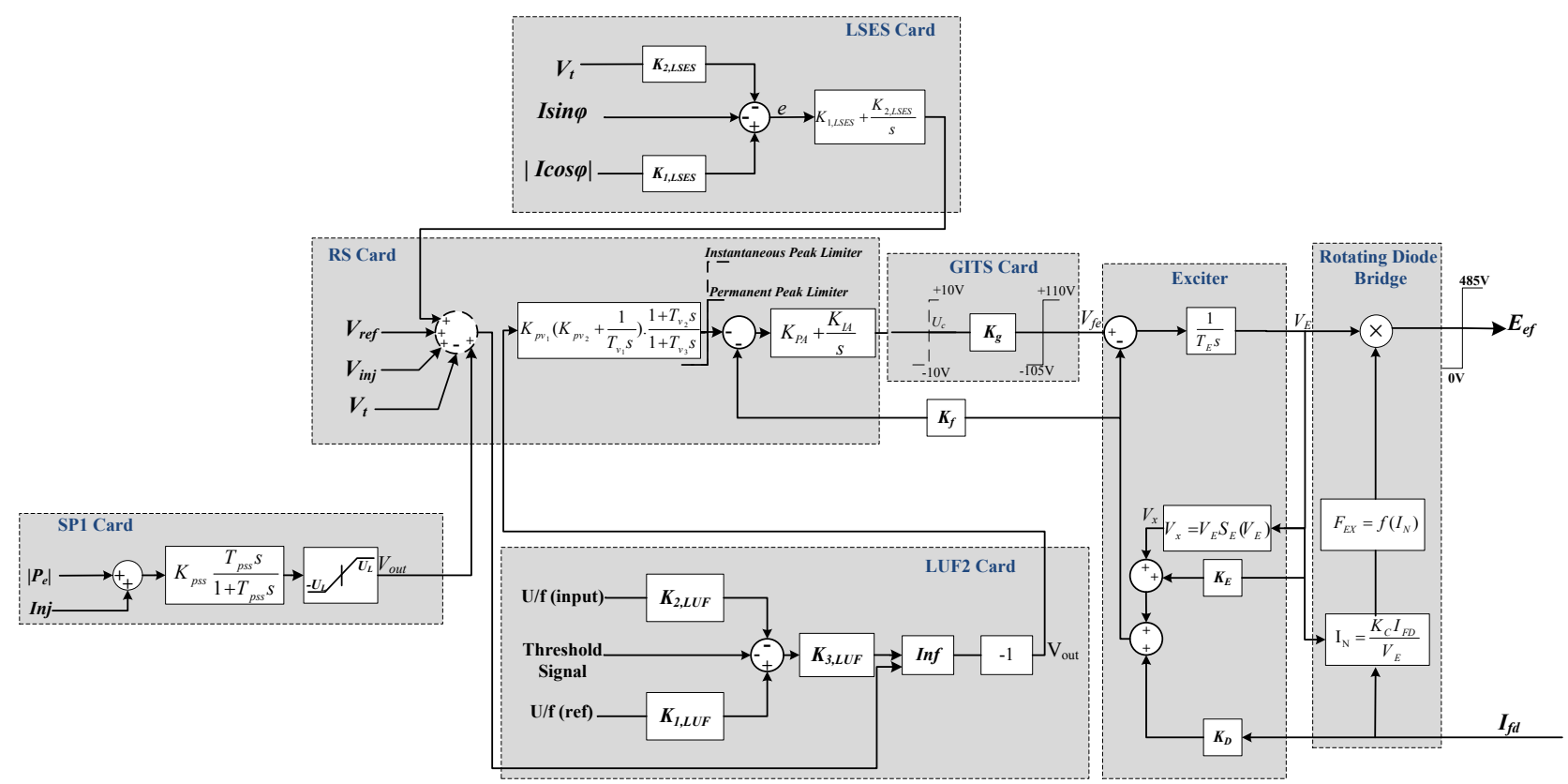

Fig. 1. The overall block diagram for modelling the excitation system

\subsection{Exciter}

In this excitation systems, the responsibility of exciter is to provide the ac voltage as the input to the rotating diode on the main shaft of synchronous machine.

3.7 Rotating Diode Bridge

The three-phase diode bridge rectifier is commonly employed to rectify the output voltage of ac exciter. The inductive effect of ac source which is modeled with inductive reactance and referred to as the commutating reactance makes a delay in the process of commutation.

3.8 Overall Model

Concatenating the aforementioned sections to a single, comprehensive model, the overall block diagram for modelling the ac excitation system in this unit will be obtained. This model is depicted in Fig. 1. The comprising parameters are identified through an optimization algorithm considering the terminal voltage as the objective function.

\section{EXPERIMENTAL VERIFICATION}

In order to verify the validity of proposed dynamical modelling and parameter estimation results, various experiments have been carried out on the $154 \mathrm{MW}$ gas power plant.

\subsection{High Active Power and Positive Reactive Power}

In this test, a $\pm 100 \mathrm{mV}$ voltage signal is injected to the AVR reference and the resulting outputs are recorded. (Fig. 2.)

4.2 High Active Power and Negative Reactive Power

Likewise, in this test, a $\pm 100 \mathrm{mV}$ voltage signal is injected to the AVR reference with different reactive power and the resulting outputs are recorded (Fig. 3.).

\section{CONCLUSIONS}

This paper presents a comprehensive modelling and identification procedure for the excitation system and synchronous generator parameters. In order to identify the dynamical parameters, a metaheuristic algorithm is employed and the terminal voltage of synchronous generator is used as the objective function. The accuracy of the proposed modelling is justified through experimental results on a real gas power plant.
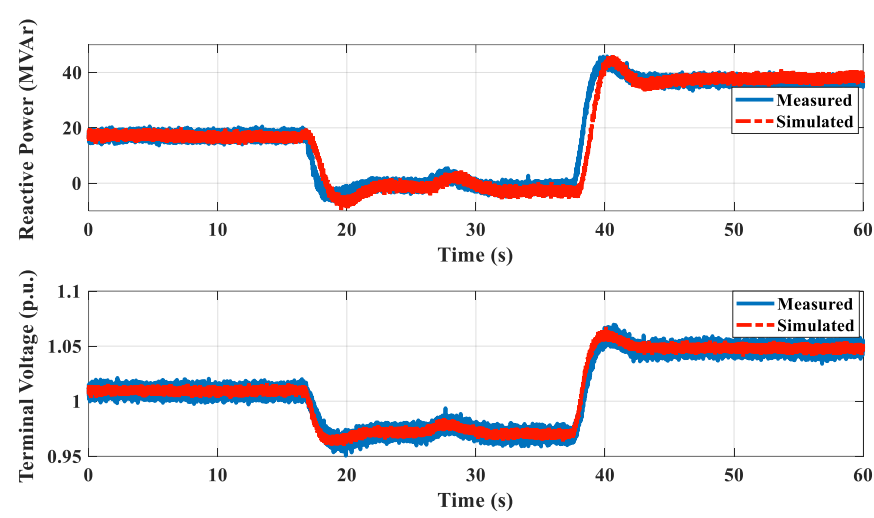

Fig. 2. The waveforms of measured and simulated signal for reactive power and terminal voltage at $\mathrm{P}=100 \mathrm{MW}$ and $\mathrm{Q}=17 \mathrm{MVAr}$.
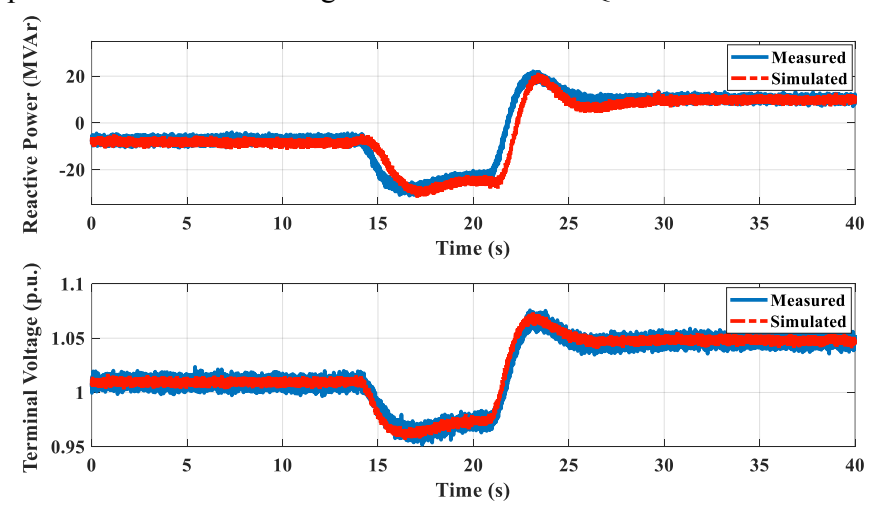

Fig. 3. The waveforms of measured and simulated signal for reactive power and terminal voltage at $\mathrm{P}=100 \mathrm{MW}$ and $\mathrm{Q}=-7.48 \mathrm{MVAr}$.

6. REFERENCES

Zaker, B. et al. (2016) 'Simultaneous Parameter Identification of Synchronous Generator and Excitation System Using Online Measurements', IEEE Transactions on Smart Grid, 7(3), pp. 1230-1238. doi:

10.1109/TSG.2015.2478971. 